\title{
Power Allocation for Detection Performance Enhancement of SEFDM Signals
}

\author{
Hedaia Ghannam and Izzat Darwazeh \\ Department of Electrical and Electronics Engineering \\ University College London, London, United Kingdom \\ E-Mail: hedaia.ghannam.15@ucl.ac.uk,i.darwazeh@ucl.ac.uk
}

\begin{abstract}
Spectrally efficient frequency division multiplexing (SEFDM) is a multi-carrier signalling format, which has a higher spectral efficiency than conventional orthogonal frequency division multiplexing (OFDM) signals. This work presents preliminary investigations into the use of power allocation for SEFDM. In this method, different subcarriers within the same SEFDM symbol are allocated different power levels. Results show that such power allocation is beneficial to SEFDM detection, with a particular case studied here of the suboptimal fixed sphere decoder (FSD) detector. The investigated method results in a drastic complexity reduction compared to FSD without power allocation for the same error performance.
\end{abstract}

\section{INTRODUCTION}

SEFDM was originally proposed in 2003 [1] and the idea behind it is to pack more subcarriers, relative to OFDM, in a given bandwidth. Orthogonality violation in SEFDM results in inter-carrier interference (ICI) and creates an environment with substantial distortion that will be severely damaging if left uncompensated.

This work, and for the first time, introduces a power allocation method to SEFDM. In this method, different subcarriers within the same SEFDM symbol are allocated with different power levels in a way similar to what has been proposed for non orthogonal multiple access (NOMA) [2]. Such power allocation is beneficial to overcome the ill-conditioned issue of SEFDM for suboptimal detectors and reduce detector complexity. For proof of concept, in this work, an FSD detector is used to detect the SEFDM signal.

Notation : Matrices are denoted by bold uppercase letters, bold lowercase letters represent column vectors. $(.)^{\mathrm{H}}$ is the Hermitian operator, $\lceil$.$\rceil is the ceiling function,$ $\lfloor$.$\rceil is the slice function and \|$.$\| is the Euclidean norm.$

\section{SEFDM Signals AND Power Allocation}

SEFDM is a multi-carrier modulation technique, where symbols are generated similarly to OFDM. The discrete SEFDM-modulated signal, $\mathbf{x} \in \mathbb{C}^{Q \times 1}$ is [3]

$$
\mathbf{x}=\frac{1}{\sqrt{Q}} \sum_{q=0}^{Q-1} \sum_{n=0}^{N-1} z_{n} \exp \left(j 2 \pi \alpha \frac{q n}{Q}\right)
$$

where $\mathbf{z} \in \mathbb{C}^{N \times 1}$ is the complex baseband symbols to be modulated by $N$ subcarriers, $Q \geq N$ is the total number of samples and $1 / \sqrt{Q}$ is employed for normalization purposes. $\alpha \in(0,1]$ is the compression factor, hence saving $(1-\alpha) \times 100 \%$ from the originally occupied bandwidth. In OFDM $\alpha$ is unity.

If the SEFDM signal in (1) were to be transmitted over additive white Gaussian noise (AWGN) (w), with zero mean and variance $\sigma_{n}^{2}=N_{0} / 2$, where $N_{0}$ is the noise power spectral density, the SEFDM-received signal $\mathbf{y} \in \mathbb{C}^{Q \times 1}$ can be presented by

$$
\mathbf{y}=\mathbf{x}+\mathbf{w} \text {. }
$$

At the receiver, the demodulated signal $\hat{z}_{n} \in \mathbb{C}$, affected by the channel and ICI is given by [3]

$$
\begin{aligned}
\hat{z}_{n} & =\frac{1}{\sqrt{Q}} \sum_{q=0}^{Q-1} y_{q} \exp \left(-j 2 \pi \alpha \frac{q n}{Q}\right), 0 \leq n \leq N-1, \\
& =\boldsymbol{\Lambda} \mathbf{z}+\mathbf{w},
\end{aligned}
$$

where $\boldsymbol{\Lambda} \in \mathbb{C}^{N \times N}$ is the correlation matrix with $\left(\Lambda_{m, n}\right)$ representing the interference from subcarrier $n$ on subcarrier $m$ and given by [4]

$$
\begin{aligned}
& \Lambda(m, n)=\exp (j \pi \alpha(m-n)) \times \\
& \exp \left(\frac{-j \pi \alpha(m-n)}{Q}\right)\left[\frac{\operatorname{sinc}(\alpha(m-n))}{\operatorname{sinc}\left(\frac{\alpha(m-n)}{Q}\right)}\right] .
\end{aligned}
$$

Unlike standard SEFDM methods, in the proposed method, subcarriers are transmitted at different power levels. The reason is that within the SEFDM symbol, there are subcarriers that can be mutually orthogonal. For $\alpha=b / c$ where $b, c \in \mathbb{Z}, b<c$ and for $c<N$, there are $\lceil N / c\rceil$ mutually orthogonal subcarriers $\left(\left|\Lambda_{m, n}\right|=0\right)$. Thus, a subset of mutually orthogonal subcarriers will be transmitted at higher power level $\left(P_{H}\right)$ and the rest of the subcarriers are allocated with the lower power level $\left(P_{L}\right)$. To have a fair comparison with SEFDM signals without power allocation, it is important to not change the total transmitted power. Thus, the increase in power level in some subcarriers results in a reduction of the power level for the rest. The ratio between the upper and lower power levels $\left(\rho=P_{H} / P_{L}\right)$ differs according to the system parameters (i.e. $N$ and $\alpha$ ) to benefit the 


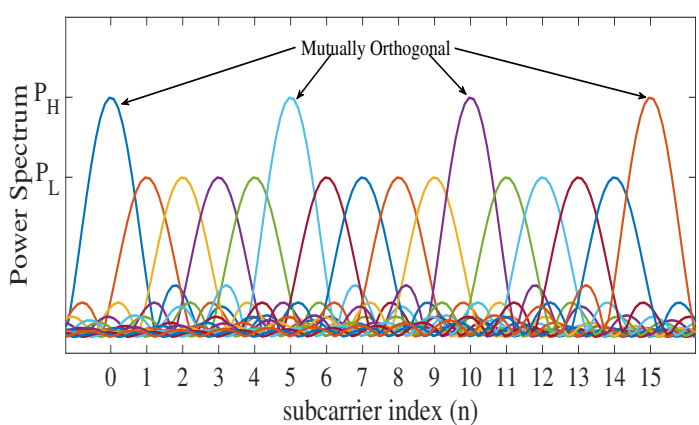

Fig. 1: SEFDM signal spectrum with power allocation for $N=16, \alpha=4 / 5, b=4$ and $c=5$.

most. In (1), the multiplication of the complex symbol $z_{n}$ by a weighting factor will result in the desired power allocation.

Fig. 1 presents the spectrum of an SEFDM symbol with power allocation for $N=16, \alpha=4 / 5$. In this case, $b=4$ and $c=5$, which results in 4 subcarriers of power $P_{H}$ and the rest of power $P_{L}$.

\section{System Design \& Results}

The output of the first stage detector $\mathbf{u} \in \mathbb{C}^{N \times 1}$ is calculated by taking a hard decision on the mutually orthogonal subcarriers with high-power level while the rest are set to zero. Thereafter, $\mathbf{u}$ is used to calculate and cancel the interference from the subcarriers with high-power level on the rest of the low-power level subcarriers, in a way similar to the method of [3], as shown in (5). The resultant vector $\mathbf{v} \in \mathbb{C}^{N \times 1}$ is the input to the second stage detector FSD.

$$
\mathbf{v}=\hat{\mathbf{z}}-\boldsymbol{\Lambda} \mathbf{u} \text {. }
$$

As a consequence of first stage interference cancellation, the correlation matrix $\boldsymbol{\Lambda}$ is updated to $\boldsymbol{\Lambda}$, where the elements referred to the interference already cancelled in the first stage is set to zero. This adjustment to $\boldsymbol{\Lambda}$ results in an orders of magnitude reduction in its condition number, which is beneficial for FSD because the initial zero forcing (ZF) estimate is crucial to its performance.

The FSD algorithm searchs within a limited subspace, where at every level $(w)$, a fixed number of nodes (tree width) $T_{w}=2^{w}$, are examined. The FSD estimate is obtained from the minimisation problem stated in [5]

$$
\hat{\mathbf{z}}_{\text {out }}=\arg \min _{\tilde{\mathbf{z}} \subset \mathcal{H}, \tilde{z} \in M}\|\mathbf{L}(\mathbf{p}-\tilde{\mathbf{z}})\|^{2} \leq \breve{g},
$$

where $\tilde{\mathbf{z}}$ is a candidate SEFDM symbol within the search subspace $\mathcal{H}, M$ is the constellation cardinality, $\mathbf{L}$ is an upper triangular matrix defined by the Cholesky decomposition as $\dot{\boldsymbol{\Lambda}}^{\mathrm{H}} \boldsymbol{\Lambda}=\mathbf{L}^{\mathrm{H}} \mathbf{L}, \mathbf{p}=\left(\hat{\boldsymbol{\Lambda}}^{\mathrm{H}} \hat{\boldsymbol{\Lambda}}\right)^{-1} \boldsymbol{\Lambda} \mathbf{v}$ and $\breve{g}$ is the radius of the search sphere, which corresponds to the distance from the ZF estimate $\hat{\mathbf{z}}_{Z F}=\left\lfloor\boldsymbol{\Lambda}^{-1} \mathbf{v}\right\rceil$. We can notice that the first stage detector affects both FSD $\mathrm{ZF}$ estimate and correlation matrix.

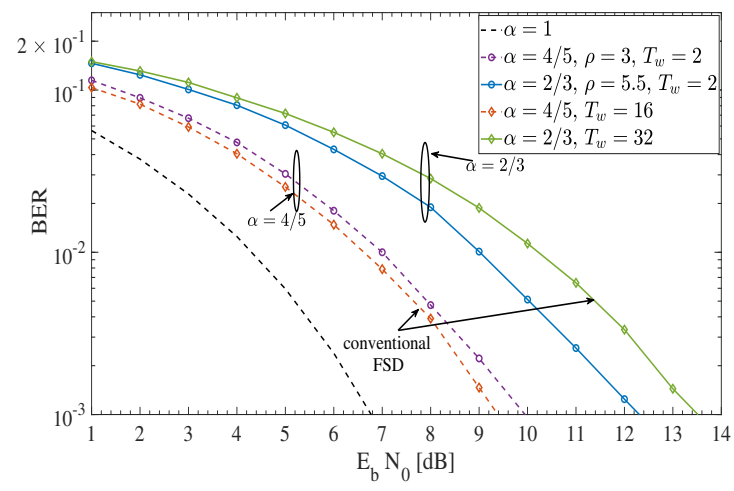

Fig. 2: SEFDM signals BER performance with $M=4$, $N=16, T_{w}=2$ and $\alpha=4 / 5,2 / 3$.

Fig. 2 shows the bit error rate (BER) performance versus energy per bit over $N_{0}\left(E_{b} / N_{0}\right)$ for the proposed method and compares it to conventional FSD. The parameters are $M=4, N=16$ and $\alpha=4 / 5,=2 / 3$. The FSD is of unity level $\left(T_{w}=2\right)$ for power allocation and it is of higher level order for conventional FSD. The power ratio $\rho$ is chosen to result in optimal BER performance, with no details given here for brevity. From the results, it can be noticed that $T_{w}$ of conventional FSD (diamond marker) needs to be increased substantially (16 and 32 for $\alpha=4 / 5$ and $\alpha=2 / 3$, respectively) compared to the power allocation method $\left(T_{w}=2\right)$ to achieve a comparable BER performance.

\section{CONCLUSion}

This work introduces the use of power allocation for SEFDM. Results show that such method is beneficial to SEFDM detection with a particular case presented here for the FSD detector. The investigated method results in a drastic complexity reduction compared to conventional FSD for the same BER performance. Further investigation is the subject of ongoing work.

\section{REFERENCES}

[1] M. Rodrigues and I. Darwazeh, "A Spectrally Efficient Frequency Division Multiplexing Based Communications System," in Proc. 8th Int. OFDM Workshop, Hamburg, Nov. 2003, pp. 48-49.

[2] L. Dai, B. Wang, Y. Yuan, S. Han, C. 1. I, and Z. Wang, "Non-orthogonal multiple access for 5G: solutions, challenges, opportunities, and future research trends," IEEE Commun. Mag., vol. 53, no. 9, pp. 74-81, September 2015.

[3] K. Park, H. Kim, A. Lee, D. Kang, and W. Oh, "Iterative frequency-domain inter-carrier interference cancellation for coded SEFDM," Electronics Letters, vol. 53, no. 19, pp. 1333-1335, September 2017.

[4] S. Isam and I. Darwazeh, "Characterizing the Intercarrier Interference of Non-orthogonal spectrally efficient FDM System," in 8th CSNDSP, 2012, July 2012, pp. 1-5.

[5] T. Xu, R. C. Grammenos, F. Marvasti, and I. Darwazeh, "An Improved Fixed Sphere Decoder Employing Soft Decision For the Detection of Non-orthogonal Signals," IEEE Commun Lett, vol. 17, no. 10, pp. 1964-1967, October 2013. 